\title{
A Cylindrical Wideband Conformal Fractal Antenna for GPS Application
}

\author{
Ratikanta Sahoo ${ }^{1}$, Damera Vakula ${ }^{1}$ \\ ${ }^{1}$ Department of ECE, National Institute of Technology, Warangal, India \\ *corresponding author, E-mail: ratikanta. sahoo15@gmail.com
}

\begin{abstract}
In this paper, a novel wideband conformal fractal antenna is proposed for GPS application. The concepts of fractal and partial ground are used in conformal antenna design for miniaturization and bandwidth enhancement. It comprises of Minkowski fractal patch on a substrate of Rogers RT/duroid 5880 with permittivity 2.2 and thickness of $0.787 \mathrm{~mm}$ with microstrip inset feed. The proposed conformal antenna has a patch dimension about $0.39 \lambda \mathrm{mm} \times 0.39 \lambda \mathrm{mm}$, and partial ground plane size is $29 \mathrm{~mm} \times 90 \mathrm{~mm}$. The proposed antenna is simulated, fabricated and measured for both planar and conformal geometry, with good agreement between measurements and simulations. The size of the fractal patch is reduced approximately by $32 \%$ as compared with conventional patch. It is observed that the conformal antenna exhibits a fractional bandwidth(for the definition of $-10 \mathrm{~dB}$ ) of $43.72 \%$ operating from 1.09 to $1.7 \mathrm{GHz}$, which is useful for L1(1.561.58GHz), L2(1.21-1.23GHz), L3(1.37-1.39GHz), L4(1.36$1.38 \mathrm{GHz})$, and $\mathrm{L} 5(1.16-1.18 \mathrm{GHz})$ in GPS and Galileo frequencies: $\mathrm{E}=1589.742 \mathrm{MHz}(4 \mathrm{MHzbandwidth}), \mathrm{E} 2=1561$.

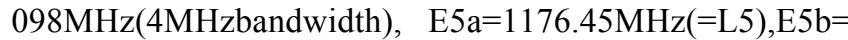
$1207.14 \mathrm{MHz}$, and $\mathrm{E} 6=1278.75 \mathrm{MHz}(40 \mathrm{MHz}$ bandwidth). The radiation pattern exhibits an omnidirectional pattern, and gain of proposed antenna is $2.3 \mathrm{dBi}$ to $3.5 \mathrm{dBi}$ within operating frequency range.
\end{abstract}

\section{Introduction}

Global Positioning System (GPS) require compact and wideband antennas for receivers installed on moving vehicles, missiles and hand held devices. These antennas play a crucial role in the GPS receiver as they have to receive weak signals from satellites and as well work in remote areas. GPS antennas are to be placed on convenient location on the platform where omnidirectional coverage is assured without any obstruction of the signal transmitted from satellites. The platform for mounting the antenna is not always flat. Some instances, it is recommended to make the antenna conformal to the structure to reduce aero dynamical drag and less visible to human eye and have omnidirectional coverage for example on missile or aircraft. There is a requirement to develop conformal antennas with omnidirectional coverage for GPS receivers. As space constraints on the vehicle are always a limitation for antenna design, a compact structure is very well required for GPS applications. So, miniaturization as well must be applied in the antenna design. Fractal geometry is one of the promising technique by which the electrical lengh of the antenna is increased with a gradual decreas in size of the antenna. Different fractal geometrie are available like Koch fractal, Minkowski fractal, Sierpensiki gasket design in published literature.

The GPS receiver is designated to operate in L1(1.561.58GHz),L2(1.21-1.23GHz), L3(1.37-1.39GHz), L4(1.36$1.38 \mathrm{GHz})$ and L5 (1.16-1.18GHz) bands, where L1 is used for $\mathrm{C} / \mathrm{A}$ acquisition by older satellites and L2 is used for $\mathrm{P}(\mathrm{Y})$ codes and military codes by newer satellites, L5 is proposed for civilian safety of life signal, L3 for nuclear detonation detection and L4 is under development for atmospheric research. Therefore, it can be considered that GPS application frequency bands extend from 1.16 to $1.58 \mathrm{GHz}$ frequency range. A single antenna which operates over the entire frequency band is often essential which can be considered as wide band antenna. The use of GPS signals at different bands helps in reducing jamming vulnerability and in improving positioning accuracy. A proper antenna design is vital for GPS applications. Microstrip antennas are best choice for conformal antennas due to their thin profile configuration, less weight and low volume. These antennas have reduced aerodynamic drag, smaller radar cross section, more angular coverage and less visible to human eye [1-3]. Such kind of antennas have always been the focus of the research, and several forms of practical antennas were reported by $\mathrm{He} \mathrm{Zhu}$ et al.[4].

Few planar antenna designs have been proposed for GPS application. Hua-Ming Chen et al. have proposed a folded square patch antenna for Global Positioning System (GPS) receivers for vehicular communication [5]. But the peak gain and fractional bandwidth are very less. Integrated Global Positioning System (GPS) and Satellite Digital Audio Radio System(SDARS) antenna are proposed for automotive use by M. M. Bilgic et al.[6]. But the antenna is not compact structure. Encheng Wang et al. have proposed a circularly polarized (CP) patch antenna using organic magnetic substrate for GPS application with less bandwidth covering only L1 frequency bands[7]. Zhang xiangjun et al. proposed a conformal cylindrical GPS antenna array for L1 band only with $20 \mathrm{MHz}$ bandwidth and less peak gain[8]. Published literature on GPS antenna on planar surfaces at 
L1, L2 and L5 frequency bands are few and operating only at either of single band. Due to the disadvantages of the planar GPS antenna presently used for GPS receivers, conformal antennas are investigated and proposed on a cylindrical surface to imitate the effect of wrapping the antenna on the surface of vehicle.

Some antennas are designed using fractal concept on flat surface at higher frequency compared with GPS frequency [9-13]. Dholakiya et al. proposed a microstrip antenna with a circular shaped fractal slot to improve the bandwidth for 2 to $6 \mathrm{GHz}$ having working bandwidth of $52 \%$ [9]. Karmakar et al. proposed a complementary stacked patch antenna with fractal shape defect for wideband characteristics for 1.8 to $6.4 \mathrm{GHz}$ with fractional bandwidth of $89.1 \%$ on flat surface [10]. In published literature [11-13] the bandwidth informations are not available. The antennas are designed on the FR4 substrate in [9-12]. The dielectric loss tangent of FR4 is 0.02 where as Rogers RT/duroid is 0.0009 which indicates that the losses of FR4 are more compared with Rogers RT/duroid 5880. Also FR4 is not flexible like Rogers RT/duroid 5880, hence RT duroid is selected in the proposed work.

In this paper, a novel high-performance wideband cylindrical conformal antenna based on the fractal structure is proposed for GPS and Galileo frequency application. The proposed cylindrical conformal antenna exhibits wideband bandwidth. The planar, as well as the proposed cylindrical conformal antenna, are designed, simulated and validated.

\section{Antenna Design}

Consider a square patch antenna as shown in Fig.1, fed by an inset microstrip line feed. The width of square patch can be calculated as follows:

$$
W=\frac{c}{2 f_{r} \sqrt{\frac{\varepsilon_{r}+1}{2}}}
$$

where $\mathrm{c}$ is the velocity of light, $f_{r}$ is the resonant frequency of the antenna and $\varepsilon_{r}$ is the relative permittivity of the substrate.

The microstrip line feed is fed at the edge, where as the inset feed is fed away from the edge to reduce the impedance. The impedance at the edge is large as current is zero, feed impedance matching can be established by properly selecting the width and depth of inset feed. A square patch antenna is designed for GPS frequency and dimensions are slightly optimized to achieve a better performance. A self symmetrical structure is used to maximize the electrical length and for reducing the size of antenna. The fractal shapes using the Minkowski loop generator are utilized for miniaturization in this paper.

The initial shape is the square patch of size W. The design modifications of Minkowski fractal antennas are shown in Fig. 2 and Fig. 3 respectively. The Minkoski fractal structure is modified in two stages and three sides are replaced with a new scaled generator. The optimized indentation factor of 1 st and 2nd stages are 0.2 and 0.25 respectively. In order to achieve wide operating bandwidth and omnidirectional coverage partial ground concept is selected. The dimensions of the proposed antenna are as follows: $\mathrm{W}=57 \mathrm{~mm}, \mathrm{~L} 1=20 \mathrm{~mm}, \mathrm{~W} 1=4 \mathrm{~mm}, \mathrm{~L} 2=39 \mathrm{~mm}$, $\mathrm{W} 2=2.4 \mathrm{~mm}, \mathrm{~L} 3=8 \mathrm{~mm}, \mathrm{~W} 3=2 \mathrm{~mm}, \mathrm{~W} 4=30 \mathrm{~mm}$. The size of partial ground is $29 \mathrm{~mm} \times 90 \mathrm{~mm}$. The substrate size is selected as $90 \mathrm{~mm} \times 100 \mathrm{~mm}$. The impedance of microstrip line is designed for $50 \Omega$.

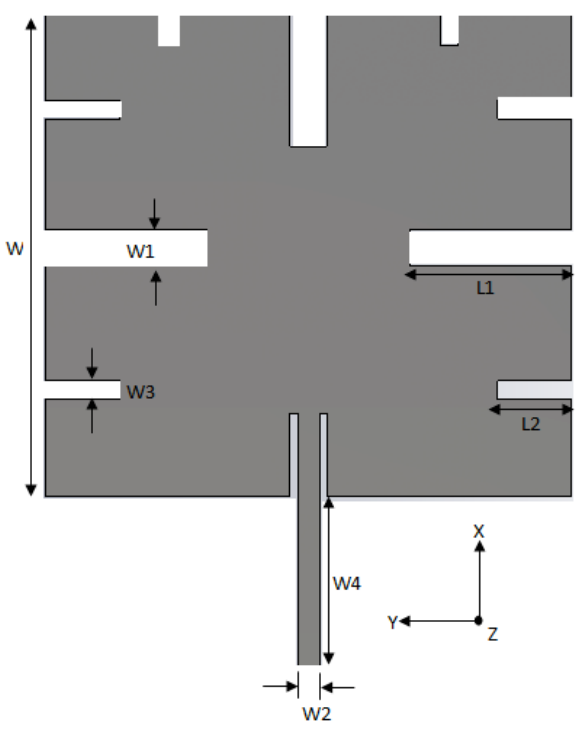

(a)

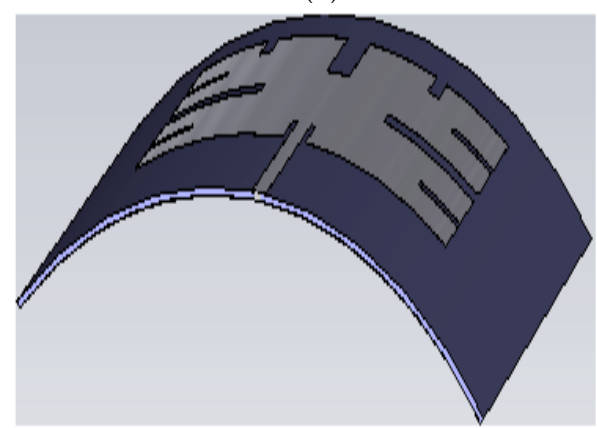

(b)

Figure 1: The top views of (a) Planar antenna (b) Conformal antenna with $60 \mathrm{~mm}$ radius of curvature

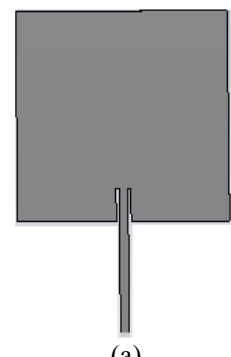

(a)

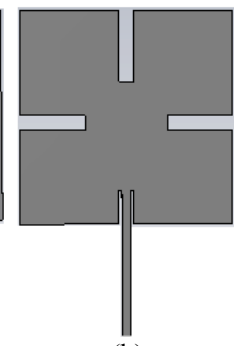

(b)

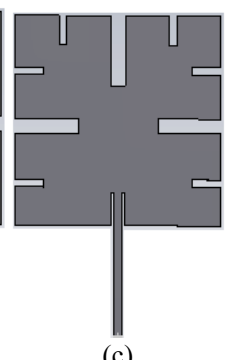

(c)
Figure 2: The Scheme of Minkowski fractal iteration of planar structure (a) Initiator(0th iteration) (b) 1st iteration (c) 2nd iteration. 


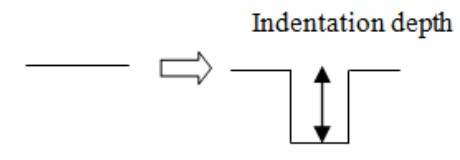

Figure 3: Minkowski fractal generator.

The cylindrical conformal antenna of $60 \mathrm{~mm}$ radius curvature is obtained by the transformation of the planar antenna. The resonant frequency of conformal antenna for the TM mode is given by[3]

$$
f_{m n}=\frac{c}{2 \sqrt{\varepsilon_{r}}}\left[\left(\frac{m}{W}\right)^{2}+\left(\frac{n}{L}\right)^{2}\right]^{1 / 2}
$$

where $W=2 R \Phi_{0}+t / \sqrt{\varepsilon}$ is the effective circumferential length of the patch and $L=Z_{m}+t / \sqrt{\varepsilon_{r}}$ is the effective axial length,' $\varepsilon_{r}$ 'is the relative permittivity and 't' is the thickness of the cylindrical substrate. The dimensions of the patch are $2 R \phi_{o}$ and $Z_{m}$, with $\mathrm{R}$ is the radius of curvature of the cylinder, $\phi_{o}$ is the angle from the centre of the patch to the straight edge and $Z_{m}$ is the axial length of the patch.

\section{Simulation Results}

The substrate is chosen to be Rogers RT/duroid 5880, which has soft property and can be easily bend to make into conformal. The electrical parameters of the material are about relative permittivity $(\mathrm{Er})$ of 2.2 , dielectric loss tangent $(\tan \delta)$ of 0.0009 and a substrate thickness of $\mathrm{h}=0.787 \mathrm{~mm}$. The proposed conformal, as well as a planar antenna are designed and simulated using CST studio suite-2016. Minkowski fractal iterations from the initiator to the second of the conducting patch are simulated for both planar and conformal with $60 \mathrm{~mm}$ radius surfaces. The concept of the partial ground is also reported in this paper. Although back radiation occurs due to truncation of ground, bandwidth is increased because of suppression of surface wave propagation.

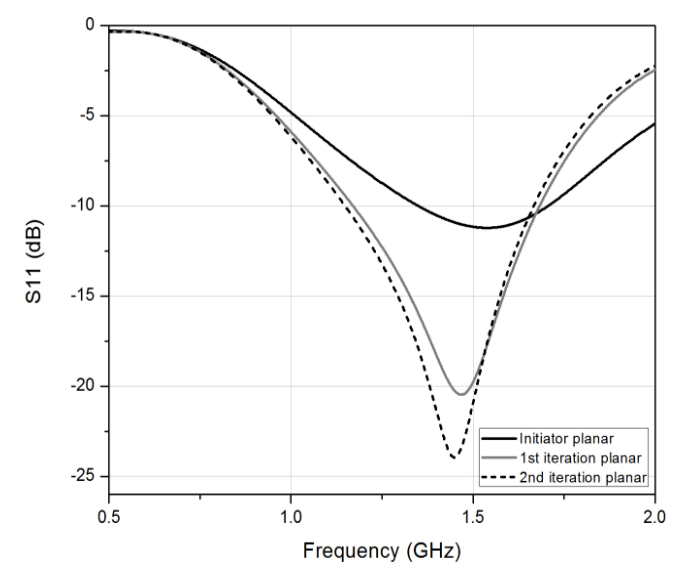

(a)

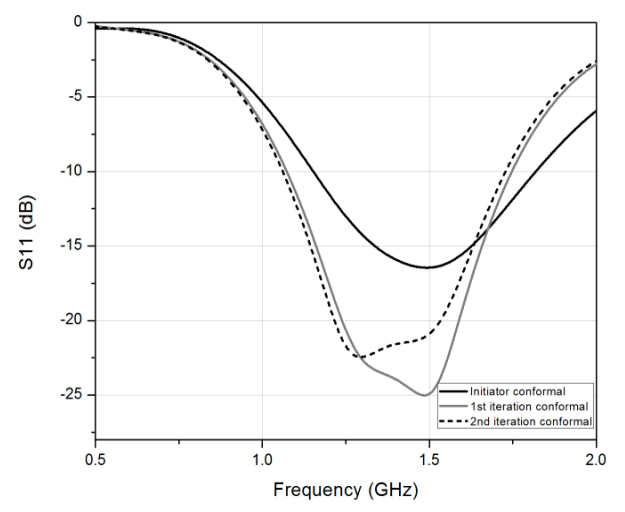

(b)

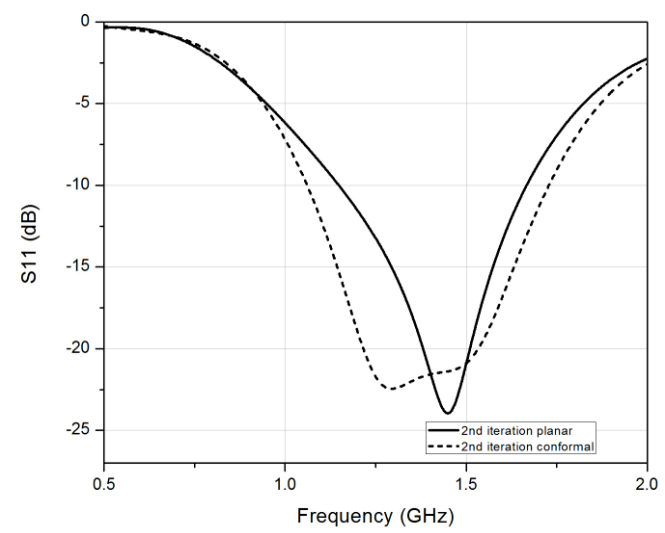

(c)

Figure 4: The simulated magnitude of the reflection coefficient.

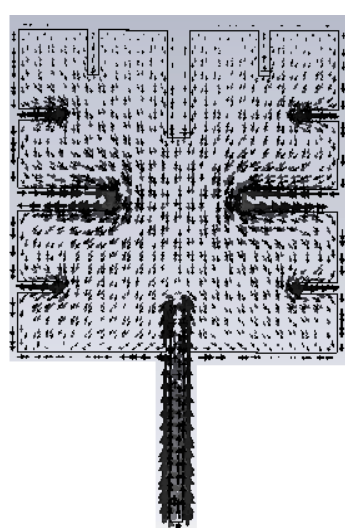

(a) (b)

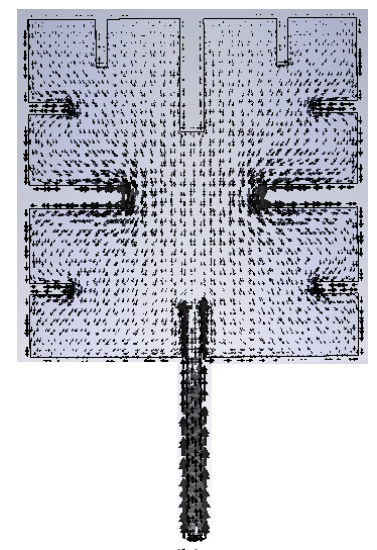

Figure 5: The simulated field distribution at $1.575 \mathrm{GHz}$ (a)2nd iteration of planar antenna (b)2nd iteration of conformal antenna with $60 \mathrm{~mm}$ radius.

The magnitude of the reflection coefficient of simulated structure of different iterations are compared in Fig.4. The operating frequency range of 0th iteration,1st iteration and 2nd iteration of Minkowski fractal planar antenna are 1.35$1.70 \mathrm{GHz}, 1.17-1.68 \mathrm{GHz}$, and $1.15-1.66 \mathrm{GHz}$ respectively. The results demonstrate that by modifying the design of minkoskwi fractal, the effective electrical length for current distributions is also increasing for which the operating frequency is shifted from right to left.

From the magnitude of the reflection coefficient plot of simulated conformal antenna, the operating frequency range 
of initiator design, 1st stage and 2nd stage of Minkowski fractal conformal antenna are $1.15-1.8 \mathrm{GHz}, 1.09-1.7 \mathrm{GHz}$, and $1.07-1.68 \mathrm{GHz}$ respectively. That means by increasing the number of iterations, the effective electrical length for current distributions is also increased for which the operating frequency is shifted from right to left as in planar structure. Also the magnitude of the reflection coefficient between planar and conformal antenna are compared in Fig.5. The operating frequency has been changed from conformal antenna to planar one. It is because of increasing the effective resonant length due to bending structure of the antenna, resulting in shifting of operating frequency.

To observe the effect of field distributions, the simulated field distributions for 2 nd iterated planar as well as conformal antennas are shown in Fig.5. There is more dense of field distributions near two sides of fractal slots. Also, the field distributions of conformal antenna is less dense as compared with planar structure, because the entire surface of the antenna is not parallel to the principal axes.

\section{Results and Discussions}

To validate the proposed antenna simulation results, the simulated 2nd stage Minkowski fractal based planar as well as cylindrical conformal with $60 \mathrm{~mm}$ radius are fabricated on $\mathrm{RT} /$ duroid 5880 substrate of volume $57 \times 57 \times 0.787 \mathrm{~mm}^{3}$ and measurements are performed. The prototype fabricated antennas are shown in Fig.6. Foam material is considered for making $60 \mathrm{~mm}$ radius of cylinder which is used for cylindrical conformal. Because it has no radiation effect of the antenna. The magnitude of the reflection coefficient for both planar and corresponding cylindrical conformal antennas are measured using an HP N5230A vector network analyzer. Fig.7 shows the measured magnitude of the reflection coefficient of the proposed antennas. The measured impedance bandwidth( $-10 \mathrm{~dB})$ magnitude of the reflection coefficient of $43.72 \%$ ranges from 1.09 to $1.7 \mathrm{GHz}$ and agrees well with the simulated results.

The radiation patterns for both planar and cylindrical conformal at different frequencies are evaluated in an anechoic chamber. Both the planar and conformal antenna have a partial ground plane. The E-plane of the radiation pattern is plotted along $\mathrm{xz}$ plane and $\mathrm{H}$-plane pattern is plotted along yz plane which is obtained as circle as illustrated in Fig. 8. By the gain transfer method, the gain of the proposed antenna is measured. The peak gain ranges from 2.3 to $3.5 \mathrm{dBi}$ in the operating frequency band(1.09 to 1.7GHz) is shown in Fig.9.

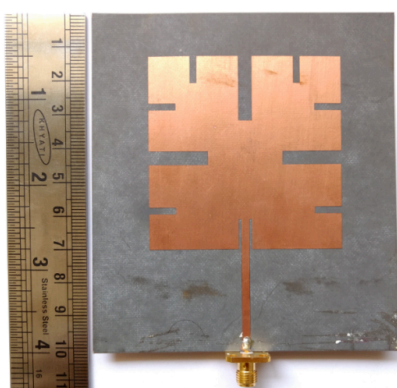

(a)

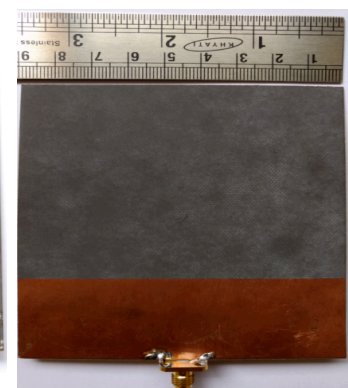

(b)

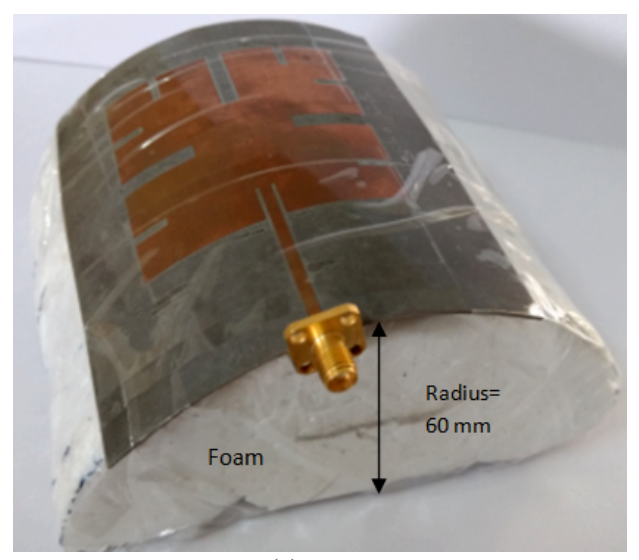

(c)

Figure 6: Photographs of fabricated antenna (a) Top view of Planar antenna (b) Bottom view of planar antenna (c) Top view of proposed conformal antenna with $60 \mathrm{~mm}$ radius of curvature.

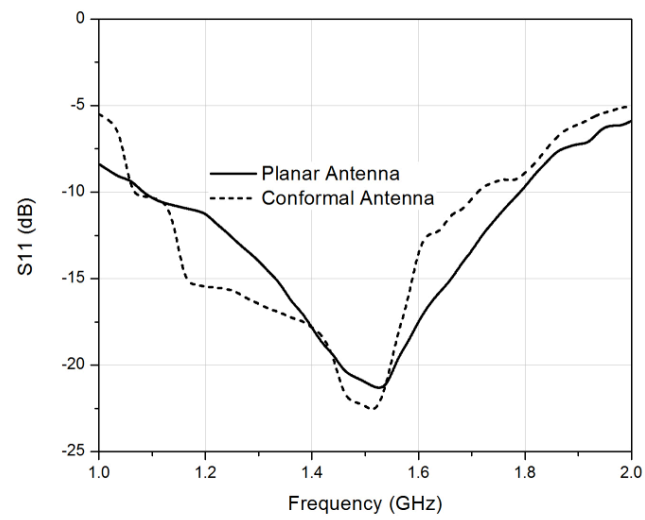

Figure 7: The measured magnitude of the reflection coefficient of planar and conformal antenna.

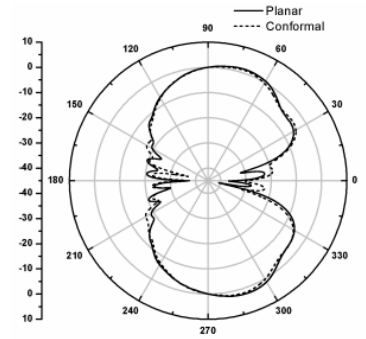

(a)

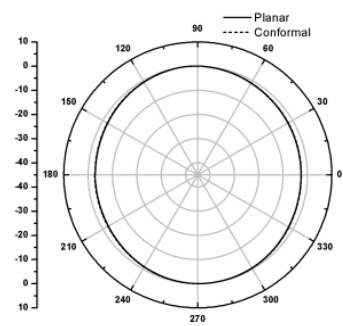

(c)

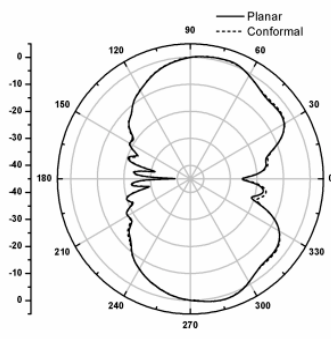

(b)

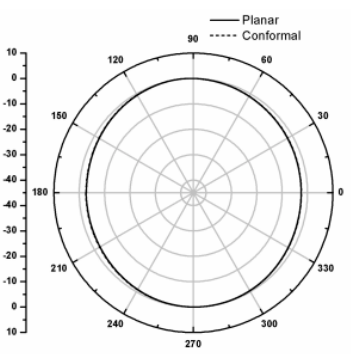

(d)
Figure 8: The measured radiation patterns of planar and conformal antenna (a) E-plane pattern at $1.5 \mathrm{GHz}$ (b) E-plane pattern at $1.57 \mathrm{GHz}$ (c) $\mathrm{H}$-plane pattern at $1.5 \mathrm{GHz}$ (d) $\mathrm{H}$ plane pattern at $1.57 \mathrm{GHz}$. 


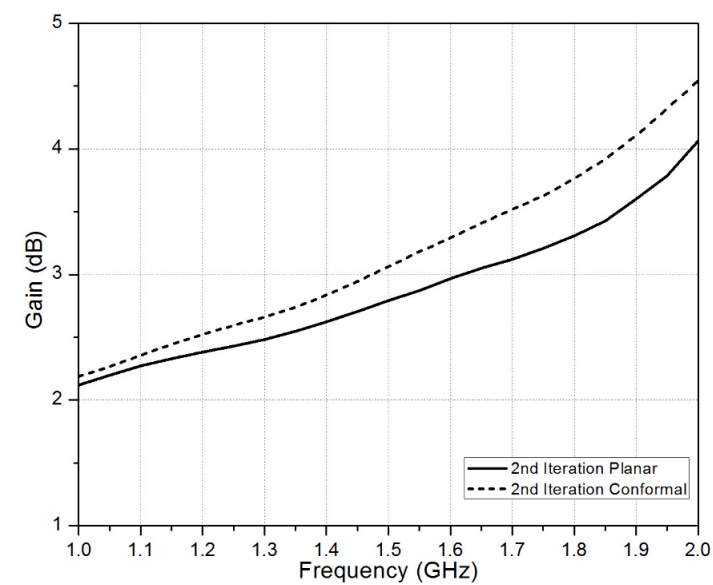

Figure 9: The measured gain plot of planar and conformal antenna.

The proposed cylindrical conformal antenna is compared with existing planar antennas at GPS frequency collected from literature survey, and it is given in Table 1.

Table 1: Comparison of the proposed cylindrical antenna with planar antenna existing in literature.

\begin{tabular}{ccccc}
\hline $\begin{array}{c}\text { anten } \\
\text { na }\end{array}$ & $\begin{array}{c}\text { size(peri } \\
\text { meter) } \\
{[\mathrm{mm}]}\end{array}$ & $\begin{array}{c}\text { frequency } \\
{[\mathrm{GHz}]}\end{array}$ & $\begin{array}{c}\text { 10dB } \\
\text { bandwidth }\end{array}$ & $\begin{array}{c}\text { gain[ } \\
\mathrm{dB}]\end{array}$ \\
\hline $\begin{array}{c}\text { Propo } \\
\text { sed }\end{array}$ & 380 & $\begin{array}{c}\text { L1,L2,L3, } \\
\text { L4,L5 } \\
\text { band }\end{array}$ & $\begin{array}{c}610 \mathrm{MHz}(4 \\
3.72 \%)\end{array}$ & 3.5 \\
work & & & \\
{$[5]$} & 314.15 & 1.575 & $2.1 \%$ & -11 \\
{$[6]$} & 395.84 & 1.575 & $20 \mathrm{MHz}$ & 6.5 \\
{$[7]$} & 240 & 1.575 & $20 \mathrm{MHz}$ & 4 \\
\hline
\end{tabular}

\section{Conclusion}

This paper presents the design of fractal based planar as well as a conformal antenna with a $60 \mathrm{~mm}$ radius of curvature. The proposed conformal antenna is compared with the existing references of the planar antenna at GPS frequency. It offers a good wideband fractional bandwidth of about $43.72 \%$ and covering the bandwidth requirements of the standard GPS and Galileo frequency application which can be used to guide the anti-tank guided missile(ATGM). Furthermore, the antenna size is reduced by $34 \%$ and has an omnidirectional radiation pattern with a gain of about $2.3 \mathrm{dBi}$ to $3.5 \mathrm{dBi}$ within the operating frequency range of $1.09 \mathrm{GHz}$ to $1.7 \mathrm{GHz}$.

\section{References}

[1] Anon., Aeronautical satellites, Aircraft Engineering and Aerospace Technology 59 (3),2-4,1987.

[2] Munson Re, Conformal microstrip antennas, Microw. J. $31(3), 1988$.

[3] L.Josefsson, P.Persson, Conformal Array Antenna Theory and Design, IEEE Press, 2006.
[4] He Zhu; Xianling Liang; Sheng Ye; Ronghong Jin;Junping Geng, A Cylindrically Conformal Array With Enhanced Axial Radiation, IEEE Antennas and Wireless Propagation Letters, 1653-1656, 2016.

[5] Hua-Ming Chen,Yi-Fang Lin, Chien-Hung Chen, Chien-Yuan Pan, And You-Shiang Cai,"Miniature Folded Patch GPS Antenna for Vehicle Communication Devices, IEEE Transactions On Antennas And Propagation, Vol.63, NO.5, 2015.

[6] M. M. Bilgic And K. Yegin," Modified Annular Ring Antenna for GPS and SDARS Automotive Applications, IEEE Antennas And Wireless Propagation Letters, Vol.15, 2016.

[7] Encheng Wang, Qiuping Liu," GPS Patch Antenna Loaded with Fractal EBG Structure Using Organic Magnetic Substrate, Progress In Electromagnetics Research Letters, Vol. 58, 23-28, 2016.

[8] Zhang Xiangjun, Ma Xiaoping, Guan Xueyuan, "Design of Cylindrical Conformal Microstrip GPS Antenna Arrays, International Conference on Microwave Technology and Computational Electromagnetics, Beijing,p.105-108, 2009.

[9] Dholakiya, Hitesh L., and Dhaval A. Pujara. "Improving the bandwidth of a microstrip antenna with a circular-shaped fractal slot." Microwave and Optical Technology Letters 55.4 (2013): 786-789.

[10]Karmakar, Anirban, et al. "Complementary stacked patch antenna with fractal shape defect for wideband characteristics." Microwave and Optical Technology Letters 56.4 (2014): 944-947.

[11] Mahatthanajatuphat, Chatree, et al. "A rhombic patch monopole antenna with modified Minkowski fractal geometry for UMTS, WLAN, and mobile WiMAX application." Progress In Electromagnetics Research 89 (2009): 57-74.

[12] Ataeiseresht, R., C. H. Ghobadi, and J. Nourinia. "A novel analysis of Minkowski fractal microstrip patch antenna." Journal of Electromagnetic Waves and Applications 20.8 (2006): 1115-1127.

[13] Manafi, Sara, and Hai Deng. "Design of a small modified Minkowski fractal antenna for passive deep brain stimulation implants." International Journal of Antennas and Propagation 2014 (2014). 